\title{
Quantitative measurement of the vibrational amplitude and phase in photorefractive time-average interferometry: A comparison with electronic speckle pattern interferometry
}

Rohleder, Henrik; Petersen, Paul Michael; Marrakchi, A.

Published in:

Journal of Applied Physics

Link to article, DOI:

10.1063/1.357063

Publication date:

1994

Document Version

Publisher's PDF, also known as Version of record

Link back to DTU Orbit

Citation (APA):

Rohleder, H., Petersen, P. M., \& Marrakchi, A. (1994). Quantitative measurement of the vibrational amplitude and phase in photorefractive time-average interferometry: A comparison with electronic speckle pattern interferometry. Journal of Applied Physics, 76(1), 81-84. https://doi.org/10.1063/1.357063

\section{General rights}

Copyright and moral rights for the publications made accessible in the public portal are retained by the authors and/or other copyright owners and it is a condition of accessing publications that users recognise and abide by the legal requirements associated with these rights.

- Users may download and print one copy of any publication from the public portal for the purpose of private study or research.

- You may not further distribute the material or use it for any profit-making activity or commercial gain

- You may freely distribute the URL identifying the publication in the public portal 


\title{
Quantitative measurement of the vibrational amplitude and phase in photorefractive time-average interferometry: A comparison with electronic speckle pattern interferometry
}

\author{
Henrik Rohleder \\ Physics Department, Technical University of Denmark, DK-2800 Lyngby, Denmark \\ Paul M. Petersen \\ Optics and Fluid Dynamics Department, P.O. Box 49, DK-4000 Roskilde, Denmark \\ A. Marrakchi \\ Siemens Corporate Research. 755 College Road East, Princeton, New Jersey 08540
}

(Received 7 September 1993; accepted for publication 10 March 1994)

\begin{abstract}
Time-average interferometry is dealt with using four-wave mixing in photorefractive $\mathrm{Bi}_{12} \mathrm{SiO}_{20}$. By introducing a proper sinusoidal phase shift in the forward pump beam it is possible to measure the amplitude and phase everywhere on a vibrating object. Quantitative measurements of the phase and amplitude of the vibrating structure are demonstrated in photorefractive time average interferometry. The photorefractive interferometer is compared with the performance of a commercial electronic speckle pattern interferometer (ESPI). It is shown that the dynamic photorefractive holographic interferometer improves the image quality considerably and is able to extend the measurable range for the acoustic vibration amplitude and frequency compared to what is obtainable with the ESPI equipment.
\end{abstract}

\section{INTRODUCTION TO TIME-AVERAGE INTERFEROMETRY IN PHOTOREFRACTIVE MATERIALS}

Time-average interferometry of vibrating diffusely scattering structures was introduced by Powell and Stetson in $1965 .{ }^{1}$ At that time they used conventional holographic recording techniques. They showed that the reconstructed interferogram is superimposed Bessel fringes that expresses the vibration amplitude. If the object is vibrating with a given frequency the intensity of the fringes will decrease as the square of the zeroth-order Bessel function. The maximum intensity fringe is situated at stationary points on the object and higher-order fringes correspond to increasing vibration amplitude. The good fringe quality in conventional holographic interferograms allows identification of higherorder fringes, which makes it possible to observe object excitations with large vibration amplitude. The method of conventional holographic recording, however, is troublesome to use in practice due to the cumbersome process of wave-front registration and film development. ${ }^{2}$

In 1971 Butters and Leendertz ${ }^{3}$ and Macovski, Ramsey, and Schaefer ${ }^{4}$ almost simultaneously introduced electronic speckle pattern interferometry (ESPI) that uses a videosystem for direct recording and display of the interferograms. With ESPI the handling and manipulation of the recorded interferograms became easier and during the last decade the technique has developed into a powerful tool for industrial nondestructive testing (NDT).$^{5-7}$ Still the image quality of the recorded interferograms is poor comparcd to conventional holography because it is necessary to place a small aperture in front of the videosystem, so that the video target is able to resolve the speckles. Because the interferograms in ESPI consist of a noisy speckle pattern only 6-8 fringes corresponding to a vibration amplitude of approximately 500 $\mathrm{nm}$ can be identified from the Bessel top. A method to extend the measurable range of vibration amplitude was demonstrated by Løkberg. ${ }^{5}$ If the reference mirror during the recording of the interferogram vibrates with the same frequency as the vibrating object, the zeroth-order Bessel fringe is displaced to areas on the object where the object and the reference mirror oscillate with the same amplitude and phase. In this way Lokberg showed that ESPI is able to measure vibration amplitudes up to $5 \mu \mathrm{m}$. With ESPI it is not possible to measure vibration amplitudes above this level, because this would result in interference fringes with a size comparable to the speckles. Due to these limitations, there has, parallel to the development of the ESPI technique, been an intensive research for producing real-time interferograms with better image quality than those obtained with TV holography used in ESPI. Dynamic holographic interferometry (DHI) in photorefractive materials has shown to be a promising candidate for improving the image quality. Timeaverage interferometry has been demonstrated in Refs. 8-11 using four-wave mixing in a photorefractive $\mathrm{Bi}_{12} \mathrm{SiO}_{20}$ (BSO) crystals. In Refs. 12 and 13 time-average interferograms are obtained by means of amplified self-diffraction in a two-beam-mixing configuration, while anisotrope selfdiffraction is used in Ref. 14. Quantitative measurements of the amplitude and phase of a vibrating structure, however, have so far not been demonstrated with DHI in photorefractive materials.

In this article we describe how the technique of reference wave modulation can be incorporated in a dynamic holographic interferometer (DHI) based on four-wave mixing in a BSO-crystal. It is shown that the Besseltop is displaced to areas on the object that oscillates with the same amplitude and phase as the reference mirror. In this way quantitative measurements of the vibration phase and amplitude can be carried out. Fringe patterns illustrating the application of this 
technique are presented, and the quality of the interferograms and the dynamic range are compared with those obtained with a commercial ESPI interferometer. Furthermore, it is shown that the solubility of the fringes are improved considerably with respect to those obtained in ESPI. It is possible to identify the position of very narrow situated fringes corresponding to strongly excited objects, so that the measurable range for the vibration amplitude can be extended. In addition the response time for the BSO crystal can be controlled with the intensity of the reference beam. At low intensity levels it is possible to examine very low vibration frequencies down to about $f_{v, \mathrm{DHI}} \approx 0.1 \mathrm{~Hz}$. ESPI is restricted by the image velocity of the camera ( 25 images/s). If we request at least 20 oscillation periods during one image recording to assure a reliable time averaging, the vibrating frequency must then exceed $f_{v \text {, ESPI }}>500 \mathrm{~Hz}$.

\section{RECORDING WITH SINUSOIDAL PHASE-SHIFTED REFERENCE BEAM}

When a sinusoidal vibrating structure with frequency $f_{v}$ is illuminated with a laser beam, the backscattered wave is phase modulated with respect to the illuminating beam. If $d_{0}(x, y)$ and $\varphi_{0}(x, y)$ are the vibration amplitude and the phase at a point $(x, y)$ on the object surface, the reflected object wave can be expressed as ${ }^{2}$

$$
\begin{aligned}
U_{0}(x, y, t)= & U_{0}(x, y) \exp \left(i \frac { 2 \pi } { \lambda } G d _ { 0 } ( x , y ) \operatorname { s i n } \left[2 \pi f_{v} t\right.\right. \\
& \left.\left.+\varphi_{0}(x, y)\right]\right)
\end{aligned}
$$

where $U_{0}(x, y)$ is the amplitude for the stationary object and $G$ is a geometrical factor that depends on the relative angles between the direction of the illuminating beam and the nor$\mathrm{mal}$ to the vibrating structure. When scattered light from oscillating points on the surface interferes in the BSO crystal with a plane reference wave they form an intensity pattern that oscillates harmonically in time and space due to the sinusoidal varying Doppler-shifled frequency. If the acoustic vibration frequency $f_{v}$ is much greater than the inverse of the response time of the crystal $\left(f_{v} \geqslant 1 / \tau\right)$ only the time-average value of the sinusoidally varying grating amplitude will be detected. As shown by Powell and Stetson, ${ }^{1}$ the diffracted image intensity $I(x, y)$ can then be expressed as

$$
I(x, y) \propto I_{0}(x, y) J_{0}^{2}\left(\frac{2 \pi}{\lambda} G d_{0}(x, y)\right),
$$

where $I_{0}(x, y)$ is the image intensity for a stationary object surface. $J_{0}$ is the zeroth-order Bessel function of first kind. The fringe intensity is then given by the square of the zerothorder Bessel function, i.e., it decreases as the vibration amplitude $d_{0}(x, y)$ increases. In Eq. (2) it is assumed that $f_{v} \gg 1 / \tau$ and consequently we neglect the effect of moving gratings in the BSO crystal. Furthermore, self-diffraction effects are neglected in Eq. (2). Self-diffraction takes place when the diffracted image intensity $I(x, y)$ becomes so large that it modifies the gratings inside the photorefractive crystal.
However, in experiments with photorefractive BSO the diffraction efficiency is typically less than $1 \%$ and consequently self-diffraction can be neglected.

Vibration amplitudes which give rise to fringes with intensity less than the noise level of the recording will not be detected. As mentioned in the introduction ESPI is for this reason not capable of resolving more than $6-8$ fringes because of speckle noise. ${ }^{5}$ The measurable range can be extended with use of a sinusoidal phase modulation of the pump beam during recording of the interferograms. This is accomplished by vibrating the reference mirror with the same frequency as the object. Equation (2), however, is only valid for recording with a stationary reference beam. We now show how the theory is modified when we introduce a sinusoidal phase shift in the forward pump beam. If the reference wave is incident at an angle $\theta_{r}$ on the vibrating reference mirror the reflected wave front can be expressed as

$$
U_{r}=u_{r} \exp \left(i \frac{2 \pi}{\lambda} \cos \left(\theta_{r}\right) d_{r} \sin \left(2 \pi f_{\nu} t+\varphi_{r}\right)\right),
$$

where $d_{r}$ and $\varphi_{r}$ are the amplitude and phase of the modulation, respectively. The diffracted intensity from the hologram can then be written as ${ }^{5}$

$$
\begin{aligned}
I(x, y)= & I_{0}(x, y) J_{0}^{2}\left(i \frac { 2 \pi } { \lambda } \left\{G^{2} d_{0}^{2}(x, y)+\cos ^{2}\left(\theta_{r}\right) d_{r}^{2}\right.\right. \\
& \left.\left.-2 G d_{0}(x, y) \cos \left(\theta_{r}\right) d_{r} \cos \left[\varphi_{0}(x, y)-\varphi_{r}\right]\right\}^{1 / 2}\right) .
\end{aligned}
$$

From Eq. (4) we can conclude that the image intensity will still be modulated by the square of the zeroth-order Bessel function, but the maximum intensity fringes are displaced to points on the object that oscillate with an amplitude and phase that make the argument of $J_{0}$ equal to zero, which happens for $d_{0}(x, y)=\cos \left(\varphi_{r}\right) d_{r} / G$ and $\varphi_{0}(x, y)=\varphi_{r}$. Therefore, by sweeping the amplitude and phase of the reference beam, and keeping track of the bright Bessel tops, it is possible to map the vibrational mode pattern of the whole surface using this "differential" technique.

\section{EXPERIMENTS}

The experimental setup is based on the four-wavemixing scheme shown in Fig. 1. The incoming light from the argon laser $(\lambda=514.5 \mathrm{~nm})$, is split into two parts by means of the beamsplitter $\mathrm{BS}_{1}$. The transmitted beam, that constitutes the reference wave $A_{1}$, is cleaned through a pinhole in the beam expander (BE). The readout beam $\mathrm{A}_{2}$ is derived from $A_{1}$ after retroreflection on a mirror placed behind the crystal. The interference between reflected light from the vibrating object (in Fig. 1 a loudspeaker) and the forward beam $A_{1}$ induces gratings in the BSO crystal. The phase conjugate interferogram (beam $A_{3}$ ) results from diffraction of the backward pump beam $A_{2}$ in the recorded gratings. The phase conjugate beam $A_{3}$ is separated by means of the beamsplitter $\mathrm{BS}_{2}$ and spatially filtered to remove noise. Finally, $\mathrm{A}_{3}$ is imaged onto a video camera for real time viewing of the vibration modes. The phase modulation of the reference beam is 


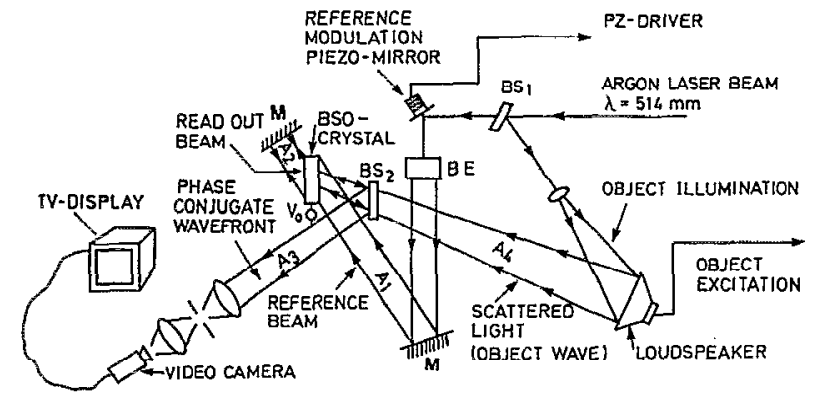

FIG. 1. Experimental setup for dynamic holographic interferometry. The basic configuration is a four-wave-mixing scheme where the time-average signal is read out as a phase conjugate interferogram. $\mathrm{BS}_{1}$ and $\mathrm{BS}_{2}$ are beamsplitters, $M$ is a mirror, and $\mathrm{BE}$ is a beam expander.

accomplished by mounting the reference mirror on a piezoelectric stack driven with a sinusoidal voltage.

The respective intensities of the various beams are $I_{1}=1.0 \mathrm{~mW} / \mathrm{cm}^{2}$ for the reference, $I_{2}=350 \mu W / \mathrm{cm}^{2}$ for the readout, and $I_{2}=40 \mu \mathrm{W} / \mathrm{cm}^{2}$ for the backscattered light. These intensities are low because of the poor reflection efficiency from the object which requires most of the power for illumination. With these intensity levels the response time of the crystal is about $1 \mathrm{~s}$. This enables us to measure vibrating frequencies down to approximately $f_{v} \approx 20 \mathrm{hz}$. With ESPI the response time is determined by the image recording velocity (25 images/s) corresponding to a response time: $\tau_{\mathrm{ESPI}} \approx 40$ $\mathrm{ms}$. To obtain reliable time average interferograms the vibration frequency then has to exceed $f_{v}>500 \mathrm{hz}$. The response time for the DHI method can be decreased further by decreasing the intensity of the reference beam or if we use a photorefractive crystal like $\mathrm{BaTiO}_{3}$ with a slower time response.

Figure 2(a) shows the phase conjugate signal originating from light scattered on the crystal from a metal plate at rest recorded with the experimental setup shown in Fig. 1. We then excite the metal plate at one of its resonance frequencies $\left(f_{v}=4270 \mathrm{hz}\right)$ and obtain the time average interferogram shown in Fig. 2(b). The bright fringe at the center of the metal plate corresponds to the zeroth-order Bessel fringe. This part of the object is at rest during the vibration and light backscattered from this area will give rise to stationary

(a)

(b)
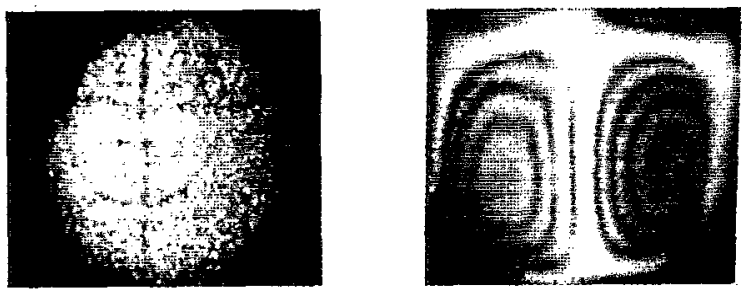

FIG. 2. Quantitative measurement of vibration amplitude and phase of a vibrating metal plate excited at $f_{v}=4270 \mathrm{~Hz}$ : (a) metal plate at rest; (b) ordinary time-average interferogram recorded with stationary reference mirror. (The mark on the metal plate is used for finding the image plane in the phase conjugate beam.) (b)

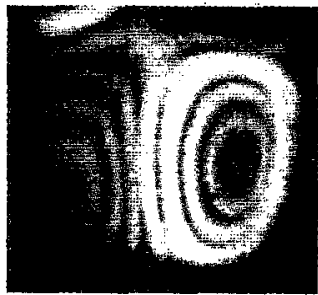

(c)

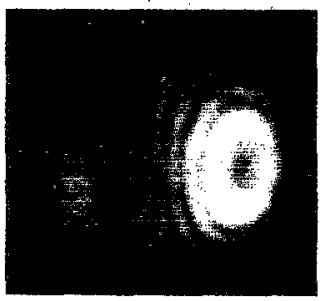

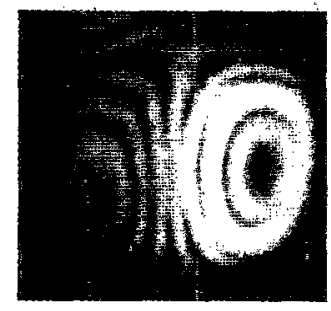

(d)

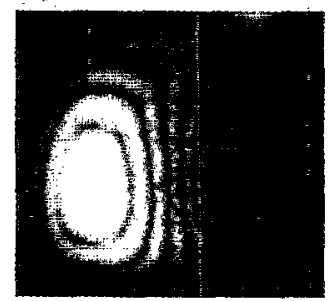

FIG. 3. (a) Modulation in phase with the upper-left- and lower-right-handside antinodes corresponding to the first-order Bessel fringe in the ordinary interferogram shown in Fig. 2(b). In (b) and (c) the vibrating phase is kept at the same value as in (a), but the vibration amplitude is increased so that the reference mirror oscillates in phase with the second and third Bessel fringes. (d) A phase shift of $180^{\circ}$ is introduced so that the modulation becomes in phase with the upper-right- and lower-left-hand-side antinodes. The amplitudes of the piezomirror are (a) $220 \mathrm{~nm}$, (b) $410 \mathrm{~nm}$, (c) $570 \mathrm{~nm}$, and (d) $570 \mathrm{~nm}$.

photoinduced gratings in the BSO crystal. Higher-order fringes correspond to increasing vibration amplitude. As the vibration amplitude increases, the contrast of the recorded fringes in the photorefractive crystal decreases and consequently the intensity in the phase conjugate output image decreases. The vibration mode is asymmetric, but this phase information is lost during recording with an unmodulated reference beam [see Eq. (2)].

Figure 3 illustrates the effect of phase modulating the reference beam. This technique allows a quantitative measurement of the vibration pattern both in amplitude and in phase. As mentioned previously, by modulating the reference beam it is possible to have the maximum of the Bessel fringes emphasize the areas of the structure under test that vibrate with the same amplitude and phase as the reference beam. These areas will now be stationary relatively to the reference beam and record a hologram with maximum diffraction efficiency. In Fig. 3(a) the amplitude of the modulation is $220 \mathrm{~nm}$, and the upper-left- and lower-right-hand antinodes of the pattern vibrate in phase with the reference mirror. Increasing the amplitude of the vibration results in other areas of the structure being highlighted. Figure 3(b) corresponds to $410 \mathrm{~nm}$ and Fig. 3(c) to $570 \mathrm{~nm}$. As an example of the effect of introducing a relative phase shift in the reference beam Fig. 3(d) shows, for the same vibrational amplitude as in Fig. 3(c), the interferogram when the reference beam has been phase shifted by $180^{\circ}$. These results show that the two oscillating modes (right- and left-hand 
(a)

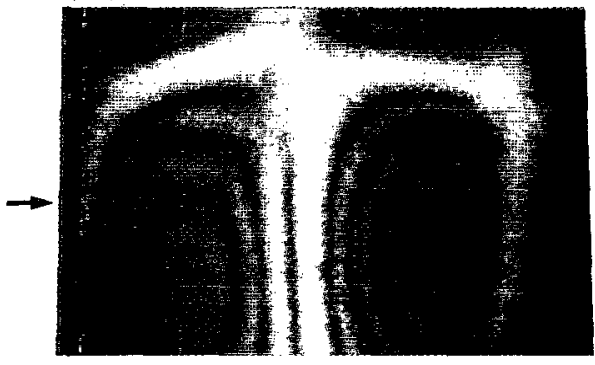

(b)

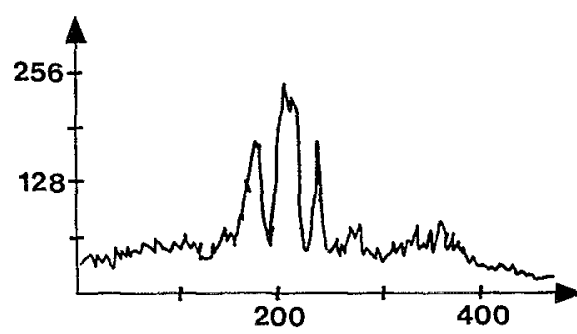

(c)

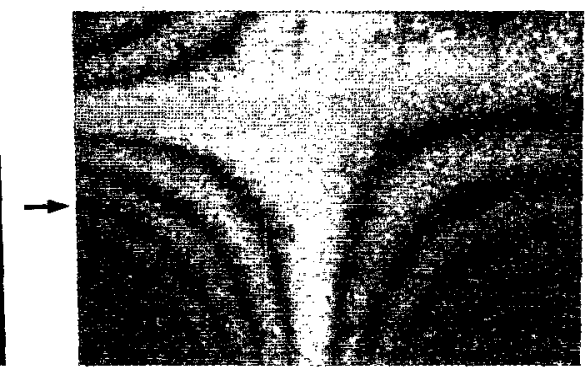

(d)

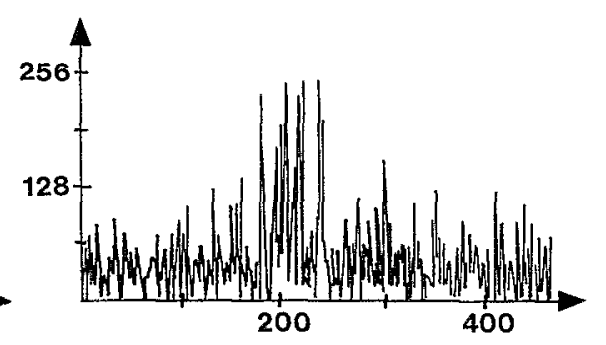

FIG. 4. (a) Time-average interferogram recorded with the DHI meter. (b) The intensity distribution through a line perpendicular to the fringes of the interferogram in (a). (c) Time-average interferogram recorded with the ESPI camera. (d) The intensity distribution through a line perpendicular to the fringes of the interferogram in (c). The arrows in (a) and (c) indicate the position at which the line scans are taken on the photographs to obtain (b) and (d).

sides of the metallic plate) are antisymmetric. In the case of a nonresonant oscillation mode the phase distribution is more complex, but the technique described above would still be applicable and would yield quantitative measurements of both the amplitude and the phase of the vibration.

The comparison between ESPI and DHI in terms of image quality is shown in Fig. 4 where we have plotted the intensity distribution across a section of the interferogram of the metallic plate shown in Fig. 2(b). Figures 4(a) and 4(b) are for the DHI method and 4(c) and 4(d) for ESPI. Figures 4(b) and 4(d) show that speckle is clearly visible in both DHI and ESPI. In ESPI the speckle is an inherent part of the interferogram, whereas in DHI the spcckle pattern is less pronounced due to the averaging effect of the photorefractive crystal: It is clear from the figures that it is possible to resolve the fringes in case of DHI but it is more difficult with ESPI because of speckle noise. Due to the high holographic quality of the gratings induced in the BSO crystal the method of DHI gives the possibility to examine objects that are excited at a relatively high excitation level where the fringe becomes very narrow. In DHI the interferogram can be further improved by using a rotating diffuser placed in the image plane.

\section{CONCLUSION}

We have shown that it is possible to carry out quantitative measurements of the phase and amplitude of vibrating structures using time average interferometry with a four- wave-mixing configuration in $\mathrm{Bi}_{12} \mathrm{SiO}_{20}$ (BSO). By modulating the phase and amplitude of the reference beam we have shown for the first time in a dynamic holographic interferometer that areas in the mode pattern can be enhanced selectively. In comparison with ESPI, DHI offers better image quality because it is not limited by speckle noise. DHI offers larger dynamic range in terms of the measurable excitation frequencies because it is possible to tailor the response time of the photorefractive crystal. Finally, DHI extends the measurable excitation amplitude because the technique does not rely on the speckle grain.

${ }^{1}$ R. L. Powell and K. A. Stetson, J. Optical Soc. Am. 55, 1593 (1965).

${ }^{2} \mathrm{R}$. Jones and C. Wykes, Holographic and Speckle Interferometry (Cambridge University Press, New York, 1989).

${ }^{3}$ J. N. Butters and J. A. Leendertz, Opt. Laser Technol. 3, 26 (1971).

${ }^{4}$ A. Macovski, S. D. Ramsey, and L. F. Schaefer, Appl. Opt. 10, 2722 (1971).

${ }^{5}$ O. J. Løkberg, J. Acoust. Soc. Am. 75, 1783 (1984).

${ }^{6}$ E. Vikhagen, Opt. Commun. 69, 4 (1989).

${ }^{7}$ P. Damgărd and M. Owner Petersen, NDT Int. 21, 422 (1988).

${ }^{8}$ J. P. Huignard, J. P. Herriau, and T. Valentin, Appl. Opt. 16, 2796 (1977).

${ }^{9}$ J. P. Huignard, J. P. Herriau, P. Aubourg, and E. Splitz, Opt. Lett. 4, 21 (1979).

${ }^{10}$ A. Marrakchi, J. P. Huignard, and J. P. Herriau, Opt. Commun. 34, 15 (1980).

${ }^{11}$ N. V. Kukhtarev and V. V. Muravev, Opt. Spectrosc. (USSR) 64, 656 (1988).

${ }^{12}$ J. P. Fuignard and A. Marrakchi, Opt. Lett. 6, 622 (1981).

${ }^{13}$ A. A. Kamshilin, E. V. Mukrushina, and M. P. Petrov, Opt. Eng. 28, 82 (1989).

${ }^{14}$ R. C. Troth and J. C. Dainty, Opt. Lett. 16, 53 (1991). 